\title{
Effect of molecular relaxation processes on travelling wave solutions of sonic boom waveforms
}

\author{
M.E. Johnson*, P.W. Hammerton \\ University of East Anglia, Norwich NR4 7TJ, UK
}

Received 16 September 2002; received in revised form 4 March 2003; accepted 26 March 2003

\begin{abstract}
Asymptotic and numerical analyses are presented for the travelling wave solution of the one-dimensional acoustic wave associated with the sonic boom, subject to thermoviscous dissipation and two molecular relaxation processes. Examination of how these relaxation processes affect the propagation of a weak shock is discussed in detail.

(C) 2003 Elsevier B.V. All rights reserved.
\end{abstract}

\section{Introduction}

Acoustics is primarily concerned with small amplitude disturbances, so non-linear effects are typically of minor significance. However, when considering the propagation of disturbances over long ranges, the cumulative effect of weak non-linearity can lead to a significant distortion of the disturbance [1]. The effect of non-linearity on finite amplitude acoustic wave propagation was studied in detail by Lighthill [2]. In particular, the competition between non-linearity and other effects was described. For one-dimensional propagation, inclusion of thermoviscosity, in addition to non-linearity leads to the well-known Burger's equation, which can be solved exactly using the Cole-Hopf linearising transformation [3,4].

While most studies of finite amplitude acoustic wave propagation have considered the effect of thermoviscosity in controlling the shock structure, in the atmosphere other physical effects may influence the shock profile. One such mechanism is molecular relaxation associated with the internal vibration of polyatomic molecules [5]. Analysis of linear propagation through a relaxing medium reveals that phase velocity increases monotonically with signal frequency, from $c_{0}$ for frequencies much less than the characteristic frequency of the relaxation mode, and tending to a limit $c_{\infty}$ for frequencies much greater than the relaxation frequency. Lighthill [2] gives general expressions for phase velocity and attenuation as a function of frequency. For propagation through the atmosphere, the relaxation modes associated with oxygen and nitrogen are significant. In this paper we consider the combined effect of these relaxation modes, thermoviscosity and non-linearity. The propagation of the disturbance can then be modelled by the augmented Burger's equation [6], together with a set of relaxation equations. Attention is restricted to one-dimensional propagation, though for the propagation of sonic booms geometric effects must also be considered.

\footnotetext{
* Corresponding author.

E-mail address: m.johnson@uea.ac.uk (M.E. Johnson).
} 
In cases where a single relaxation mode is believed to be dominant, Blythe [7], and Ockendon and Spence [8] obtained travelling wave solutions, albeit in an implicit form. This analysis showed that for some range of parameter values, a single relaxation mode alone is insufficient to support the shock and a narrow sub-shock arises controlled by other mechanisms. The two cases are known as fully-dispersed and partly-dispersed, respectively. The important feature of a partly-dispersed shock is the appearance of a finer length scale within the shock. Since the subjective annoyance associated with sonic booms is related to shock widths (or equivalently shock rise-times) the identification of the transition between the two types of shock structure is obviously important.

Although there has been some numerical investigation [9] concerning the presence of two relaxation modes, there has been no analysis on their effect on shock structure. In the presence of more than one relaxation mode, exact travelling wave solutions are not available, and progress can only be made through a combination of asymptotic and numerical schemes. Pierce and Kang [10] presented numerical data for a travelling wave through a medium with two relaxation modes and thermoviscosity, but only for one set of parameter values. Moreover, the structure of the shock was not discussed. Hence, in this paper we discuss how the structure of the shock depends on the relaxation parameters.

In Section 2 a non-dimensional equation is obtained governing one-dimensional non-linear propagation through a medium with $N$ relaxation modes and with thermoviscous effects included. Each relaxation mode is characterised by two parameters, $\tau_{i}$ a dimensionless relaxation time, and $\Delta_{i}$ the non-dimensional change in linear sound speed between low- and high-frequency signals. For propagation through air, two relaxation modes are present with relaxation times differing by two orders of magnitude. Hence attention is restricted to travelling wave solutions controlled by two relaxation modes together with viscous diffusion. In Section 3, an asymptotic analysis is presented based on the limit $\delta \ll \tau_{2} \ll \tau_{1}$, which is relevant to propagation through air. Here $\delta$ is the coefficient of thermoviscous diffusion. It is found that the shock structure can take three possible forms depending on the values of the relaxation parameters $\Delta_{1}$ and $\Delta_{2}$. In Section 4 a numerical method is described using a fourth-order Runge-Kutta numerical scheme with perturbation techniques used to obtain boundary conditions far away from the shock. Numerical results are then compared with asymptotic results, with attention focused on the case $\Delta_{1}+\Delta_{2}<1 / 2$, where asymptotic theory predicts the most intricate shock structure.

\section{Formulation}

The main equations considered in this paper can be obtained from Pierce [6]. The dimensional equations are known as the augmented Burger's equation and the relaxation equations

$$
\frac{\partial \tilde{p}}{\partial \tilde{t}}+c \frac{\partial \tilde{p}}{\partial \tilde{x}}+\left(\frac{\beta \tilde{p}}{c \rho_{0}}\right) \frac{\partial \tilde{p}}{\partial \tilde{x}}+\sum_{i=1}^{n}(\widetilde{\Delta c})_{i} \frac{\partial \tilde{p}_{i}}{\partial \tilde{x}}=\tilde{\delta} \frac{\partial^{2} \tilde{p}}{\partial^{2} \tilde{x}}, \quad\left(1+\tilde{\tau}_{i} \frac{\partial}{\partial \tilde{t}}\right) \tilde{p}_{i}=\tilde{\tau}_{i} \frac{\partial \tilde{p}}{\partial \tilde{t}}, \quad i=1,2
$$

Here $\tilde{p}$ is the acoustic pressure, $c$ the low frequency linear sound speed, $\rho_{0}$ the air density, $\tilde{\delta}$ the thermoviscous coefficient and $\beta=(\gamma+1) / 2$, where $\gamma$ is the ratio of specific heats. The quantities $\tilde{p}_{i}$, can be considered as partial pressures associated with each relaxation mode. Also associated with each relaxation mode are two parameters, $(\widetilde{\Delta c})_{i}$, the increment in sound speed due to the relevant relaxation process and $\tilde{\tau}_{i}$, the relaxation times.

The set of equations (1), can be put into non-dimensional form using the following substitutions:

$$
t=\frac{\tilde{t}}{t_{\mathrm{ref}}}, \quad x=\frac{\tilde{x}}{c t_{\mathrm{ref}}}, \quad \hat{\Delta}_{i}=\frac{(\widetilde{\Delta c})_{i}}{c}, \quad \hat{\tau}_{i}=\frac{\tilde{\tau}_{i}}{t_{\mathrm{ref}}}, \quad \hat{\delta}=\frac{\tilde{\delta}}{t_{\mathrm{ref}} c^{2}}, \quad \hat{p}=\frac{\tilde{p} \beta}{c^{2} \rho_{0}},
$$

where $t_{\mathrm{ref}}$ is some chosen reference time. This gives the following set of dimensionless equations:

$$
\frac{\partial \hat{p}}{\partial t}+\frac{\partial \hat{p}}{\partial x}+\hat{p} \frac{\partial \hat{p}}{\partial x}+\sum_{i} \hat{\Delta}_{i} \frac{\partial \hat{p}_{i}}{\partial x}=\hat{\delta} \frac{\partial^{2} \hat{p}}{\partial^{2} x}, \quad\left(1+\hat{\tau}_{i} \frac{\partial}{\partial t}\right) \hat{p}_{i}=\hat{\tau}_{i} \frac{\partial \hat{p}}{\partial t} .
$$


In the remainder of this paper, we restrict ourselves to the case of the travelling wave solution of this set of equations (3), for a pressure pulse, $\hat{p} \rightarrow P_{\mathrm{sh}}$, as $x \rightarrow-\infty$, and $\hat{p} \rightarrow 0$, as $x \rightarrow \infty$, where $P_{\mathrm{sh}}$ is the non-dimensional shock overpressure.

Making the substitution $\xi=x-V t$, and integrating once, (3) becomes

$$
(1-V) \hat{p}+\frac{\hat{p}^{2}}{2}-\hat{\delta} \frac{\mathrm{d} \hat{p}}{\mathrm{~d} \xi}+\sum_{i} \hat{\Delta}_{i} \hat{p}_{i}=\text { constant }, \quad\left(1-V \hat{\tau}_{i} \frac{\mathrm{d}}{\mathrm{d} \xi}\right) \hat{p}_{i}=-V \hat{\tau}_{i} \frac{\mathrm{d} \hat{p}}{\mathrm{~d} \xi}
$$

with boundary conditions governing (4):

$$
\begin{aligned}
& \hat{p}, \frac{\mathrm{d} \hat{p}}{\mathrm{~d} \xi}, \hat{p}_{i} \rightarrow 0 \quad \text { as } \xi \rightarrow \infty, \\
& \hat{p} \rightarrow P_{\text {sh }}, \quad \frac{\mathrm{d} \hat{p}}{\mathrm{~d} \xi}, \hat{p}_{i}, \frac{\mathrm{d} \hat{p}_{i}}{\mathrm{~d} \xi} \rightarrow 0 \quad \text { as } \xi \rightarrow-\infty .
\end{aligned}
$$

The first set of boundary conditions imply the constant in Eq. (4) is zero. The second set, when applied to (4), can be used to derive an expression for the shock speed,

$$
V=\frac{1}{2}\left(P_{\mathrm{sh}}\right)+1
$$

The parameters $V$ and $P_{\mathrm{sh}}$ can then be eliminated from the governing equations and boundary conditions by rescaling,

$$
p=\frac{\hat{p}}{P_{\mathrm{sh}}}, \quad \Delta_{i}=\frac{\hat{\Delta}_{i}}{P_{\mathrm{sh}}}, \quad \tau_{i}=V \hat{\tau}_{i}, \quad \delta=\frac{\hat{\delta}}{P_{\mathrm{sh}}} .
$$

In the remainder of this paper, only two relaxation modes are considered, in which case, (4) can be combined to give

$$
\left(1-\tau_{1} \frac{\mathrm{d}}{\mathrm{d} \xi}\right)\left(1-\tau_{2} \frac{\mathrm{d}}{\mathrm{d} \xi}\right)\left(\frac{p}{2}(p-1)-\delta \frac{\mathrm{d} p}{\mathrm{~d} \xi}\right)=\left(\tau_{1} \Delta_{1}+\tau_{2} \Delta_{2}\right) \frac{\mathrm{d} p}{\mathrm{~d} \xi}-\tau_{1} \tau_{2}\left(\Delta_{1}+\Delta_{2}\right) \frac{\mathrm{d}^{2} p}{\mathrm{~d} \xi^{2}}
$$

with boundary conditions, $p \rightarrow 1$ as $\xi \rightarrow-\infty$, and $p \rightarrow 0$ as $\xi \rightarrow \infty$.

For a single relaxation mode, exact solutions (albeit implicit), can be obtained [7,8]. For two modes, no such solutions are available. Numerical solutions of (9), are discussed in Section 4. However, for acoustic propagation through air, relaxation modes associated with nitrogen and oxygen are dominant, with relaxation times differing by two orders of magnitude. Hence, an asymptotic analysis of the travelling wave solution is possible.

\section{Asymptotic analysis}

In this section we consider the case $\delta \ll \tau_{2} \ll \tau_{1}$. By neglecting the small terms of $\mathrm{O}(\delta)$ and $\mathrm{O}\left(\tau_{2}\right)$, Eq. (9) becomes

$$
\frac{p}{2}(p-1)=\tau_{1}\left(p-\frac{1}{2}+\Delta_{1}\right) \frac{\mathrm{d} p}{\mathrm{~d} \xi}
$$

This solution has length scale $\mathrm{O}\left(\tau_{1}\right)$, hence, we define

$$
X_{1}=\frac{\xi-\xi_{1}}{\tau_{1}}
$$

where $\xi_{1}$ will be determined later in the analysis. An exact, implicit solution for $p\left(X_{1}\right)$ can be obtained,

$$
X_{1}=\left(1-2 \Delta_{1}\right) \log p+\left(1+2 \Delta_{1}\right) \log (1-p),
$$

which satisfies the boundary condition, $\xi \rightarrow-\infty$ as $p \rightarrow 1$. However, as $p \rightarrow 0, \xi \rightarrow-\infty \operatorname{sgn}\left(1-2 \Delta_{1}\right)$, so the form of the solution depends on whether $\Delta_{1}$ is less than or greater than $1 / 2$. 


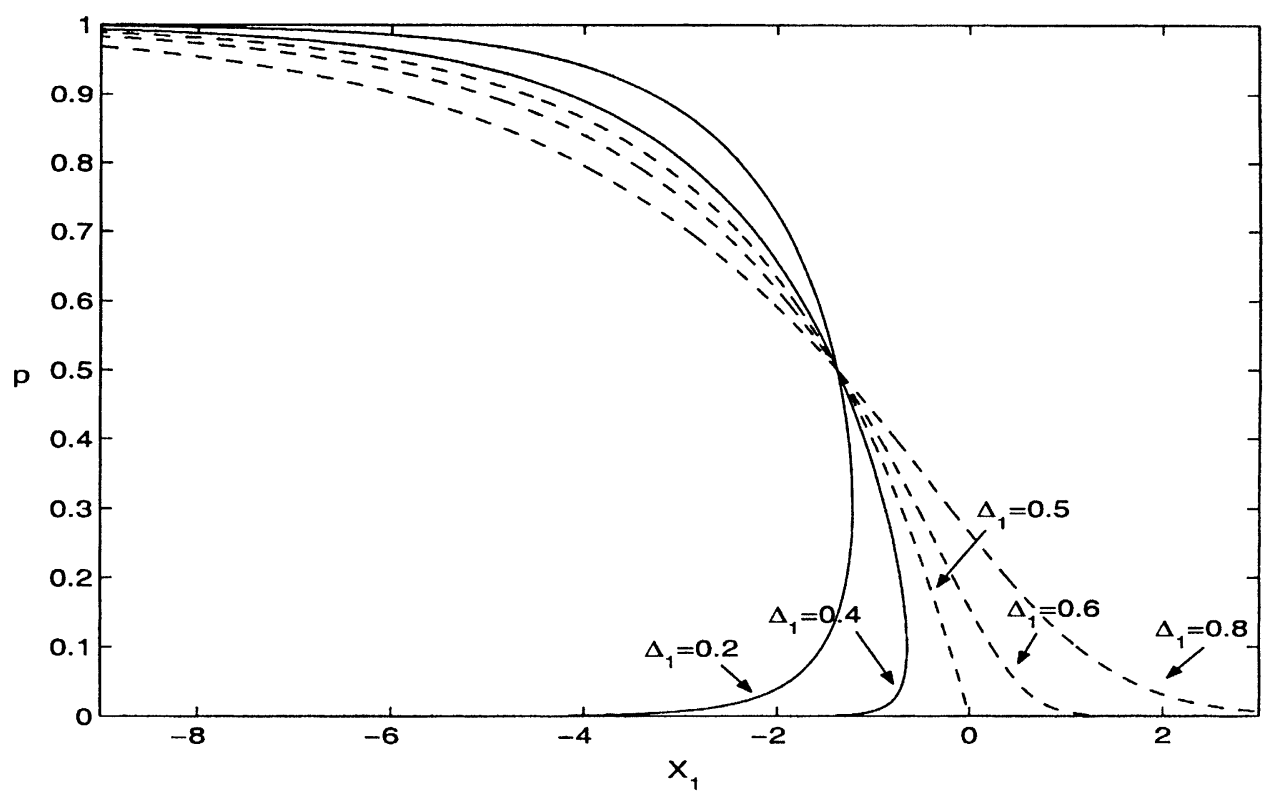

Fig. 1. Diagram shows the leading order solution (12). Solutions are shown for fully-dispersed relaxation mode, $\Delta_{1} \geq 1 / 2$ (the dotted lines), and partly-dispersed $\Delta_{1}<1 / 2$.

\section{1. $\Delta_{1}>1 / 2$}

If $\Delta_{1}>1 / 2$, then the implicit solution (12) has $p \rightarrow 0$ as $\xi \rightarrow \infty$, and hence, the waveform remains single-valued in $p$. This is known as the fully-dispersed solution and represents a physically realistic solution for the pressure $p\left(X_{1}\right)$. Thus, in this case, the viscosity and second relaxation mode have little effect on the shape of the waveform. The dashed lines in Fig. 1, represent fully-dispersed examples of solution (12).

When $\Delta_{1}=1 / 2$ the solution has a discontinuity in gradient at $p=0$. If $\Delta_{1}$ falls below the critical value, $1 / 2$, the boundary condition, $p \rightarrow 0$, as $\xi \rightarrow \infty$ is no longer satisfied and $p\left(X_{1}\right)$ is now multi-valued, as can be seen in Fig. 1. Hence (12) is not a leading order solution of (9) for all $\xi$. So, for $\Delta_{1}<1 / 2$, the first relaxation mode is insufficient to support the shock and the waveform is known as partly-dispersed. To determine the waveform for all $\xi$, the $\mathrm{O}(\delta)$ and $\mathrm{O}\left(\tau_{2}\right)$ correction terms must be considered.

\section{2. $\Delta_{1}<1 / 2, \Delta_{2}>\left(1 / 2-\Delta_{1}\right)$}

When $\Delta_{1}<1 / 2$ and the waveform is partly-dispersed, we expect the effect of the second relaxation mode to become significant at some point in the waveform. Neglecting $\mathrm{O}(\delta)$ terms, but now retaining $\mathrm{O}\left(\tau_{2}\right)$ terms, Eq. (9) becomes

$$
\tau_{1} \tau_{2} \frac{\mathrm{d}^{2} p}{\mathrm{~d} \xi^{2}}\left(p+\Delta_{1}+\Delta_{2}-\frac{1}{2}\right)+\frac{p}{2}(p-1)=\frac{\mathrm{d} p}{\mathrm{~d} \xi}\left(\left(\tau_{1}+\tau_{2}\right)\left(p-\frac{1}{2}\right)+\left(\tau_{1} \Delta_{1}+\tau_{2} \Delta_{2}\right)-\tau_{1} \tau_{2} \frac{\mathrm{d} p}{\mathrm{~d} \xi}\right)
$$

For $\Delta_{1}<1 / 2$, we expect a relatively narrow inner region, width $\mathrm{O}\left(\tau_{2}\right)$ to arise, and hence we define

$$
X_{2}=\frac{\xi-\xi_{2}}{\tau_{2}},
$$


where $\xi_{2}$ will be determined later in the analysis, which gives

$$
\tau_{1} \frac{\mathrm{d}^{2} p}{\mathrm{~d} X_{2}^{2}}\left(p+\Delta_{1}+\Delta_{2}-\frac{1}{2}\right)+\tau_{2} \frac{p}{2}(p-1)=\frac{\mathrm{d} p}{\mathrm{~d} X_{2}}\left(\left(\tau_{1}+\tau_{2}\right)\left(p-\frac{1}{2}\right)+\left(\tau_{1} \Delta_{1}+\tau_{2} \Delta_{2}\right)-\tau_{1} \frac{\mathrm{d} p}{\mathrm{~d} X_{2}}\right) .
$$

Making the expansion $p=p_{0}+\tau_{2} p_{1}+\mathrm{O}\left(\tau_{2}^{2}\right)$, the leading order equation, after integration with respect to $X_{2}$, becomes

$$
\frac{\mathrm{d} p_{0}}{\mathrm{~d} X_{2}}=\frac{p_{0}\left(p_{0}+2 \Delta_{1}-1\right)}{2\left(p_{0}+\Delta_{1}+\Delta_{2}-1 / 2\right)}
$$

using the boundary condition $\mathrm{d} p_{0} / \mathrm{d} X_{2} \rightarrow 0$, as $p_{0} \rightarrow 0$. Eq. (16) has an exact, implicit solution of

$$
X_{2}=\left(1-\frac{2 \Delta_{2}}{1-2 \Delta_{1}}\right) \log p_{0}+\left(1+\frac{2 \Delta_{2}}{1-2 \Delta_{1}}\right) \log \left(1-2 \Delta_{1}-p_{0}\right)
$$

Note that as we have expanded the region of rapid pressure transition by our shock width, $\tau_{2}$, we can consider the limits $X_{2} \rightarrow \pm \infty$, for fully dispersed solutions, as being at the two ends of this shock. Here, the boundary conditions are $p \rightarrow P_{0}=1-2 \Delta_{1}$, as $X_{2} \rightarrow-\infty$, and $p \rightarrow 0$, as $X_{2} \rightarrow \infty$.

The inner relaxation shock governed by the second relaxation mode can be matched to the outer solution governed by the first relaxation mode by considering the boundary condition for $X_{2} \rightarrow-\infty$. However, just as the first relaxation shock can be fully-dispersed or partly-dispersed, depending on the value of $\Delta_{1}$, this inner shock can also be fully or partly-dispersed.

Letting $p_{0} \rightarrow 0, X_{2} \rightarrow-\infty \operatorname{sgn}\left(1-2 \Delta_{1}-2 \Delta_{2}\right)$, and hence if

$$
\Delta_{1}+\Delta_{2}>\frac{1}{2}
$$

the inner shock is fully-dispersed, and the entire waveform consists of a partly-dispersed shock governed by the first relaxation mode, with a narrow, embedded fully-dispersed shock, governed by the second relaxation mode. Viscosity has only a small effect on the solution. Diagrammatic evidence of this is discussed later using numerical techniques from the next section.

However, if $\Delta_{1}+\Delta_{2}<1 / 2$, the second relaxation mode is insufficient to support the pressure transition from $p=P_{0}$ to $p=0$, and hence, correction terms of $\mathrm{O}(\delta)$ must be considered.

\section{3. $\Delta_{1}+\Delta_{2}<1 / 2$}

For $\Delta_{1}+\Delta_{2}<1 / 2$, the first relaxation mode is partly-dispersed, as is the inner solution controlled by the second relaxation mode. Hence, we anticipate the appearance of a narrower inner region controlled by viscosity. We now define

$$
X_{3}=\frac{\xi-\xi_{3}}{\delta}
$$

where $\xi_{3}$ will be determined in later analysis, and (9) now becomes

$$
\left(\delta-\tau_{1} \frac{\mathrm{d}}{\mathrm{d} X_{3}}\right)\left(\delta-\tau_{2} \frac{\mathrm{d}}{\mathrm{d} X_{3}}\right)\left(\frac{p}{2}(p-1)-\frac{\mathrm{d} p}{\mathrm{~d} X_{3}}\right)=\delta\left(\tau_{1} \Delta_{1}+\tau_{2} \Delta_{2}\right) \frac{\mathrm{d} p}{\mathrm{~d} X_{3}}-\tau_{1} \tau_{2}\left(\Delta_{1}+\Delta_{2}\right) \frac{\mathrm{d}^{2} p}{\mathrm{~d} X_{3}^{2}} .
$$

Making the expansion $p=\hat{p}_{0}+\delta \hat{p}_{1}+\mathrm{O}\left(\delta^{2}\right)$, the equation to leading order becomes

$$
\frac{\mathrm{d}^{2}}{\mathrm{~d} X_{3}^{2}}\left(\frac{\hat{p}_{0}}{2}\left(\hat{p}_{0}-1\right)-\frac{\mathrm{d} \hat{p}_{0}}{\mathrm{~d} X_{3}}\right)=-\left(\Delta_{1}+\Delta_{2}\right) \frac{\mathrm{d}^{2} \hat{p}_{0}}{\mathrm{~d} X_{3}^{2}}
$$


which can be integrated twice with respect to $X_{3}$ to give

$$
\frac{\mathrm{d} \hat{p}_{0}}{\mathrm{~d} X_{3}}=\frac{\hat{p}_{0}}{2}\left(\hat{p}_{0}-P_{1}\right), \quad P_{1}=1-2\left(\Delta_{1}+\Delta_{2}\right),
$$

where the constants of integration are zero from the boundary condition, $X_{3} \rightarrow \infty, \hat{p}_{0}, \mathrm{~d} \hat{p}_{0} / \mathrm{d} X_{3} \rightarrow 0$. This has exact solution

$$
X_{3}=-\frac{2}{P_{1}}\left(\log \hat{p}_{0}-\log \left(P_{1}-\hat{p}_{0}\right)\right),
$$

which can be re-arranged to give

$$
\hat{p}_{0}=\frac{P_{1}}{2}\left[1-\tanh \left(P_{1} \frac{\xi-\xi_{3}}{4 \delta}\right)\right] .
$$

From this explicit solution, it can readily be seen that the width of this viscous sub-shock region is of $\mathrm{O}\left(\delta / P_{1}\right)$.

Thus, if $\Delta_{1}+\Delta_{2}<1 / 2$, the waveform consists of a partly-dispersed shock governed by the first relaxation mode, with a narrow embedded partly-dispersed shock governed by the second relaxation mode, and finally, a narrower, embedded, fully-dispersed sub-shock controlled by the viscosity parameter. A complete diagrammatic breakdown of this waveform is provided, using numerical and asymptotic results, in Section 5.

The values of the constants $\xi_{1}$ and $\xi_{2}$ are determined in terms of $\xi_{3}$ by matching. To centre the viscous sub-shock region at the point $\xi=0$, we take $\xi_{3}=0$. As $X_{3} \rightarrow-\infty, \hat{p}_{0} \rightarrow P_{1}$, and hence, the partly-dispersed inner solution (17), controlled by the second relaxation mode, matches at leading order to the viscous solution, if $\xi_{2}$ is given by

$$
\xi_{2}=-\tau_{2}\left[\left(1-\frac{2 \Delta_{2}}{1-2 \Delta_{1}}\right) \log P_{1}+\left(1+\frac{2 \Delta_{2}}{1-2 \Delta_{1}}\right) \log \left(1-2 \Delta_{1}-P_{1}\right)\right] .
$$

Similarly, for the inner solution governed by the second relaxation mode, as $X_{2} \rightarrow-\infty, p_{0} \rightarrow P_{0}=1-2 \Delta_{1}$, and hence, matches to the partly-dispersed outer solution, governed by the first relaxation mode, if $\xi_{1}$ is given by

$$
\xi_{1}=-\tau_{1}\left[\left(1-2 \Delta_{1}\right) \log P_{0}+\left(1+2 \Delta_{1}\right) \log \left(1-P_{0}\right)\right] .
$$

For the case $\Delta_{1}<1 / 2, \Delta_{1}+\Delta_{2}>1 / 2$, where the viscous sub-shock is absent, the fully-dispersed shock associated with the second relaxation mode (17), is centred in amplitude at $\xi=0$ by choosing

$$
\frac{\xi_{2}}{\tau_{2}}=\left(\frac{2 \Delta_{2}}{1-2 \Delta_{1}}-1\right) \log \frac{P_{0}}{2}-\left(1+\frac{2 \Delta_{2}}{1-2 \Delta_{1}}\right) \log \left(1-2 \Delta_{1}-\frac{P_{0}}{2}\right) .
$$

The first relaxation shock (12) then matches at leading order to this solution using (26).

\section{Numerics}

The asymptotic structure of the travelling wave solution derived in Section 3 can be verified by comparison with numerical solutions. To solve the third-order ODE, (9), numerically, three boundary conditions are required at one end of the spatial range. To obtain these boundary conditions, we consider a perturbation analysis for $p(\xi)$, as $\xi \rightarrow \infty$. For $\xi \rightarrow \infty, p(\xi) \rightarrow 0$, and hence we write and linearise Eq. (9), using

$$
p(\xi)=0+g(\xi), \quad|g(\xi)| \ll 1, \quad \xi \gg 1,
$$

to give

$$
\delta \tau_{1} \tau_{2} \frac{\mathrm{d}^{3} g}{\mathrm{~d} \xi^{3}}-\left[\delta\left(\tau_{1}+\tau_{2}\right)+\tau_{1} \tau_{2}\left(\Delta_{1}+\Delta_{2}-\frac{1}{2}\right)\right] \frac{\mathrm{d}^{2} g}{\mathrm{~d} \xi^{2}}-\left[\frac{1}{2}\left(\tau_{1}+\tau_{2}\right)-\left(\Delta_{1} \tau_{1}+\Delta_{2} \tau_{2}-\delta\right)\right] \frac{\mathrm{d} g}{\mathrm{~d} \xi}+\frac{g}{2}=0 .
$$


We look for solutions of the form $g \propto \mathrm{e}^{-\lambda \xi}, \lambda>0$, where $\lambda$ is given by

$$
\delta \lambda+\Delta_{1} \frac{\tau_{1} \lambda}{1+\tau_{1} \lambda}+\Delta_{2} \frac{\tau_{2} \lambda}{1+\tau_{2} \lambda}=\frac{1}{2} .
$$

As $\tau_{i} \lambda /\left(1+\tau_{i} \lambda\right)$ increases monotonically, for increasing positive $\lambda$, the left-hand side of (30) increases monotonically, from 0 to $\infty$, as $\lambda$ increases from 0 to $\infty$. Therefore, we have only one real positive root, $\lambda_{1}$ say, of the equation. This positive root is found numerically by solving Eq. (30) using a Newton-Raphson method.

Since $p \sim A \mathrm{e}^{-\lambda_{1} \xi}$, as $\xi \rightarrow \infty$, we take $\xi=X_{0}, X_{0} \gg 1$, so the approximate boundary conditions on $p$ and its derivatives, at $\xi=X_{0}$ are

$$
p\left(X_{0}\right)=\epsilon, \quad p^{\prime}\left(X_{0}\right)=-\lambda_{1} \epsilon, \quad p^{\prime \prime}\left(X_{0}\right)=\lambda_{1}^{2} \epsilon, \quad \epsilon \ll 1,
$$

where ' denotes differentiation with respect to $\xi$. A fourth-order Runge-Kutta numerical technique is then used to march $\xi$ in the negative direction, from $X_{0}$. The reason for considering the large $\xi$ form of $p$ and then marching in the negative direction is that as $\xi \rightarrow-\infty$, writing $p \sim 1+A \mathrm{e}^{\lambda \xi}$, gives two positive eigenvalues for $\lambda$, and hence, conditions on $p, p^{\prime}, p^{\prime \prime}$ are not uniquely determined at $\xi=-X_{0}$.

Although this paper only addresses the case of two relaxation processes, a simple proof shows that similar numerical techniques can be used for $N>2$ such processes. The set of equations (4), with $N$ relaxation processes can be combined to give an $(N+1)$ th order equation. Far from the shock $p \rightarrow 0$, as $\xi \rightarrow \infty$, so we can linearise the equation for large, positive $\xi$, by approximating $p(\xi) \approx 0+g(\xi)$.

By looking for solutions $g \propto \mathrm{e}^{-\lambda \xi}$, this linear equation gives

$$
\delta \lambda+\sum_{N} \Delta_{N} \frac{\tau_{N} \lambda}{1+\tau_{N} \lambda}=\frac{1}{2}
$$

This equation has $N+1$ roots, but only one will be positive as, for increasing, positive $\lambda$, the left-hand side of the equation is again positive and monotonically increasing.

As we have only one positive root, we can obtain the $N+1$ boundary conditions we require for large, positive $\xi$, to solve the equation numerically.

\section{Results}

The numerical scheme described in the previous section is valid for the general case of $N$ relaxation modes, and for arbitrary values of the relaxation parameters, $\tau_{i}$ and $\Delta_{i}$, and the parameter of thermoviscous diffusion, $\delta$. Here we consider numerical solutions only for the case of two relaxation modes, with $\delta \ll \tau_{2} \ll \tau_{1}$, and compare them with asymptotic solutions derived in Section 3. From the asymptotic analysis, the most intricate shock structure arises when $\Delta_{1}+\Delta_{2}<1 / 2$. Hence the numerical solution was obtained for the parameter values

$$
\tau_{1}=1.0, \quad \tau_{2}=0.01, \quad \delta=5 \times 10^{-5}, \quad \Delta_{1}=0.1, \quad \Delta_{2}=0.2 .
$$

This is plotted as the solid line in Fig. 2. For these parameter values, the asymptotic analysis predicts that the travelling wave solution consists of three regions, each with different length scales.

\subsection{Region $A$}

Far upstream from the shock, the first relaxation mode dominates the waveform, and hence this region has width of $\mathrm{O}\left(\tau_{1}\right)$. This region can be seen in Fig. 2(a), where the solid line is the numerical solution and the dashed line marks the asymptotic solution (12), corresponding to the partly-dispersed solution for the first relaxation mode. For $1>p>P_{0}=1-2 \Delta_{1}$, good agreement is seen between the two solutions. The numerical scheme has been fixed so the centre of the viscous sub-shock is at $\xi=0$. Moving downstream, the solutions begin to separate as $p$ approaches 


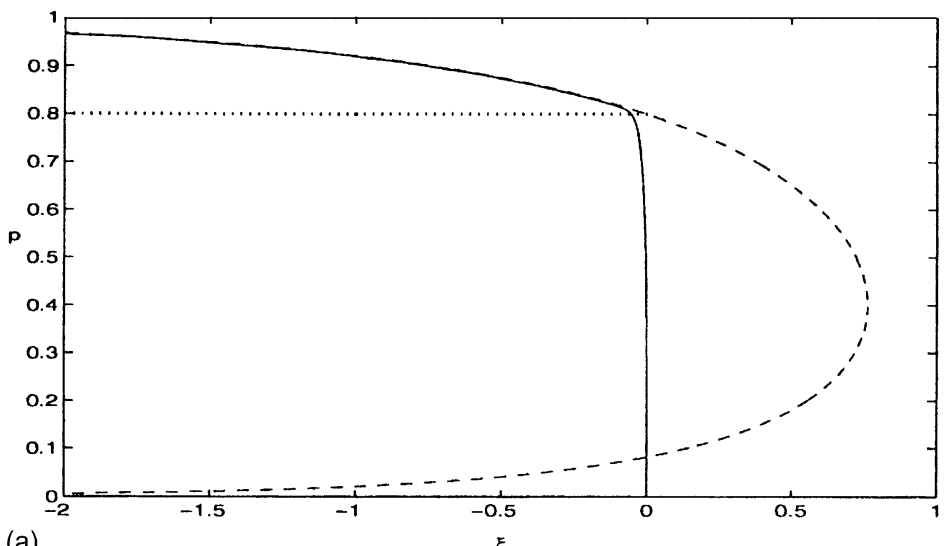

(a)
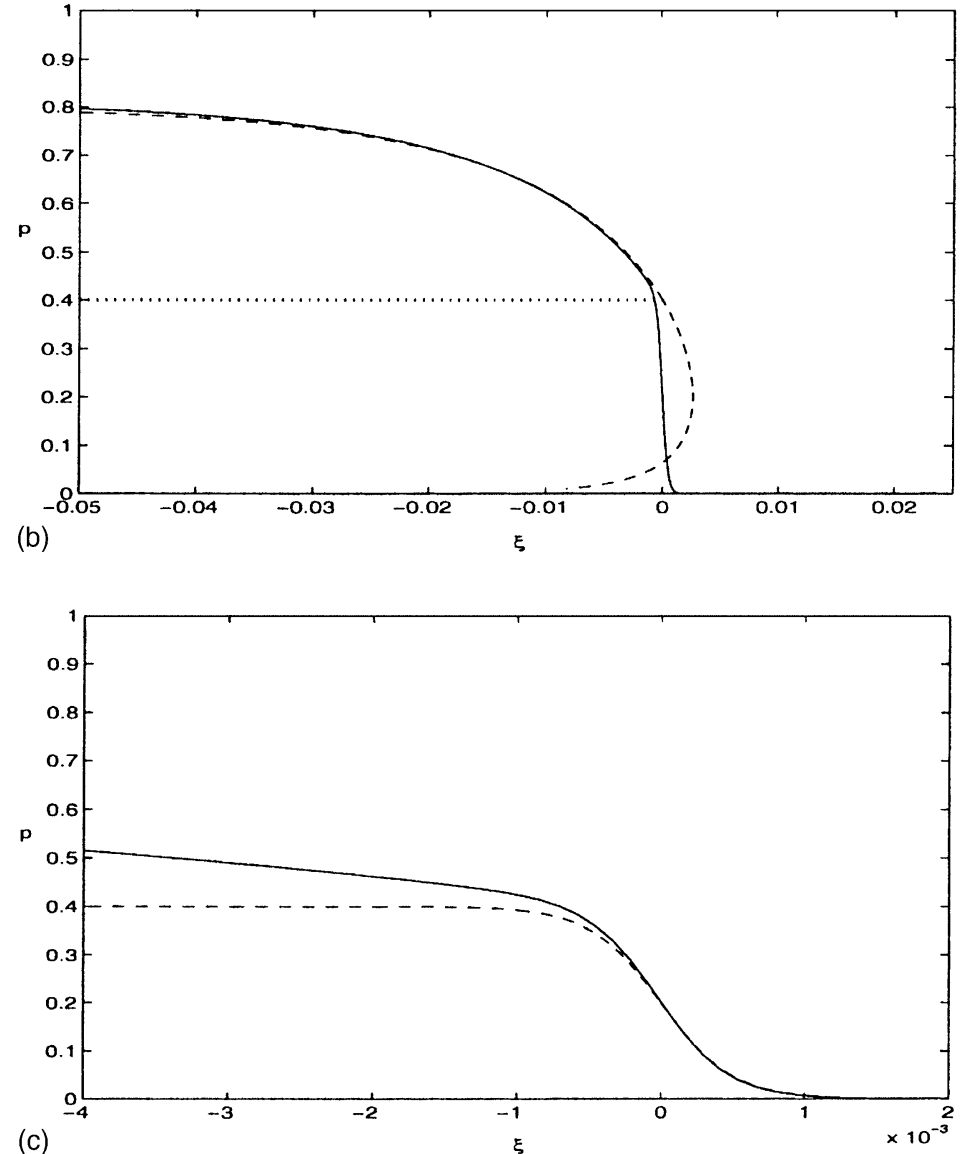

Fig. 2. Comparison of numerical and asymptotic solutions of (9), for the case $\Delta_{1}=0.1, \tau_{1}=1, \Delta_{2}=0.2, \tau_{2}=0.01$, and $\delta=5 \times 10^{-5}$. 
the value $P_{0}=0.8$, marked in Fig. 2(a) by a dotted line. This is the position at which the partly-dispersed first relaxation mode matches onto the inner solution governed by the second relaxation mode (see Section 3.2).

\subsection{Region $B$}

The middle part of the waveform is dominated by the second relaxation mode and has width of $\mathrm{O}\left(\tau_{2}\right)$. The region governs the shape of the waveform between $P_{0}=1-2 \Delta_{1}$ and $P_{1}=1-2\left(\Delta_{1}+\Delta_{2}\right)$ discussed in Sections 3.2 and 3.3, respectively. Fig. 2(b) shows this region of the waveform, which is of scale $\tau_{2}$, where the partly-dispersed inner solution (17), represented by the dashed line, is compared with the numerical solution of (9). Good agreement is seen between the numerical and asymptotic solutions for $P_{0}>p>P_{1}$. As $p$ approaches $P_{1}$ (marked by a dotted line in Fig. 2(b)), the partly-dispersed shock governed by the second relaxation mode matches a viscous sub-shock of amplitude $P_{1}$.

\subsection{Region $C$}

For the parameter values considered, asymptotic theory predicts that the head of the shock is dominated by the small viscosity parameter, and has width of $\mathrm{O}(\delta)$. This region of viscous sub-shock can be seen clearly in Fig. 2(c), which is of scale $\delta$. Good agreement is seen between the numerical solution (solid line) and the asymptotic solution (24) for the viscous shock (dotted line) for $P_{1}>p>0$.

\section{Summary}

The asymptotic analysis presented in Section 3 shows that in the limit $\delta \ll \tau_{2} \ll \tau_{1}$, the structure of the travelling shock can be divided into three classes depending on the values of the relaxation parameters $\Delta_{1}$ and $\Delta_{2}$. Numerical solutions corresponding to the three separate cases are illustrated in Fig. 3. When $\Delta_{1}>1 / 2$ (Case I), the first relaxation mode controls the shock everywhere. For $\Delta_{1}<1 / 2$ and $\Delta_{1}+\Delta_{2}>1 / 2$ (Case II) a narrow sub-shock of amplitude $1-2 \Delta_{1}$ and relative width $\tau_{2} / \tau_{1}$ controlled by the second relaxation mode is inserted in the first

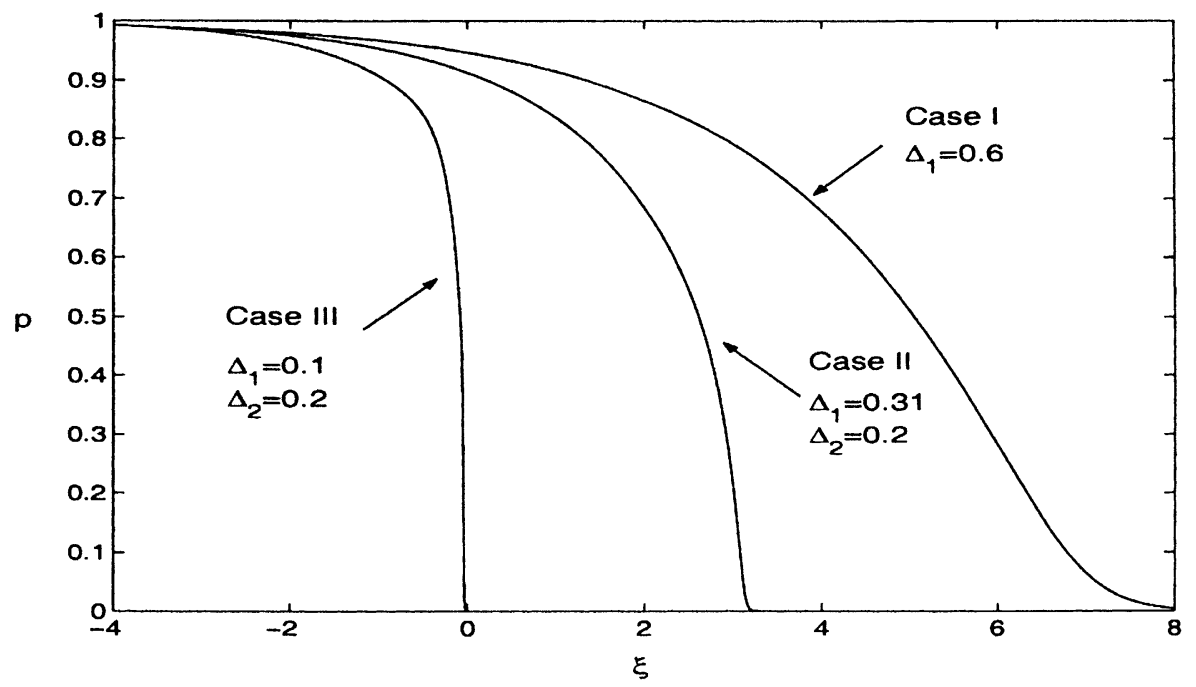

Fig. 3. Diagram shows how fully-dispersed solution is replaced with shock and sub-shocks as $\Delta_{1}$ is decreased below critical values. In this numerical example, the values $\Delta_{2}=0.2, \tau_{1}=1, \tau_{2}=0.1$ and $\delta=0.001$. 


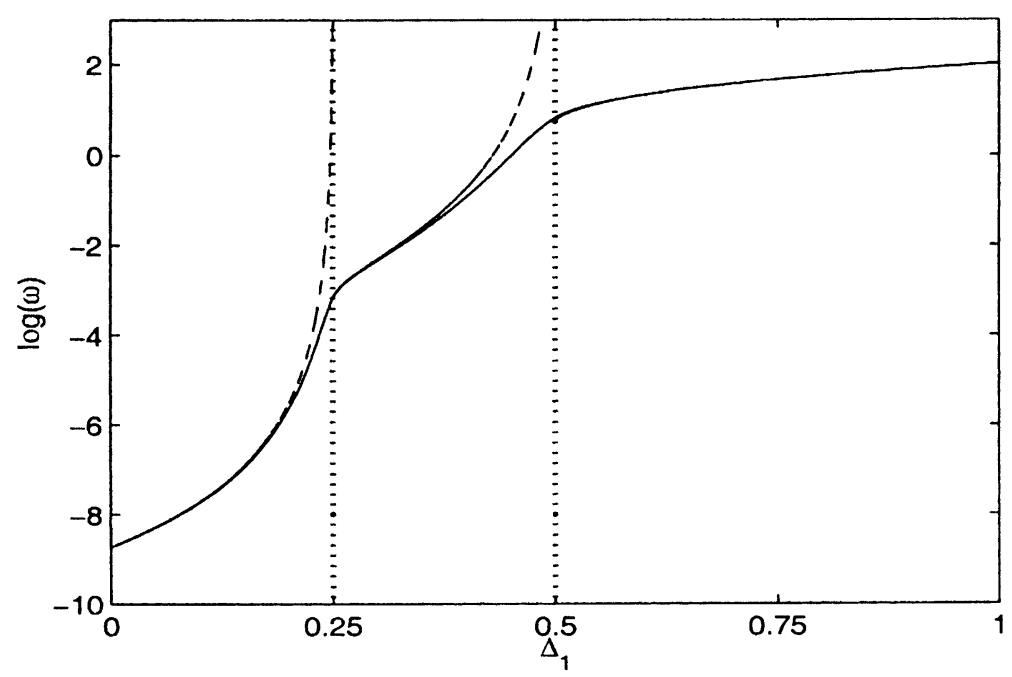

Fig. 4. Plot of shock width, $w$, on a logarithmic scale as a function of $\Delta_{1}$, for $\Delta_{2}=0.25, \tau_{1}=1, \tau_{2}=0.01$ and $\delta=5 \times 10^{-6}$.

relaxation shock. Finally, if $\Delta_{1}<1 / 2$ and $\Delta_{1}+\Delta_{2}<1 / 2$ (Case III) the shock consists of an outer partly-dispersed solution controlled by the first relaxation mode, with an inserted partly-dispersed solution of amplitude $1-2 \Delta_{1}$ and relative width $\tau_{2} / \tau_{1}$ controlled by the second relaxation mode, and finally an inner viscous shock of amplitude $1-2\left(\Delta_{1}+\Delta_{2}\right)$ and relative width $\delta / \tau_{1}$. This was the case discussed in detail in Section 5. Though Fig. 3 shows the general change in shock structure as $\Delta_{1}$ changes, namely an increase in shock slope as $\Delta_{1}$ decreases, the detailed structure cannot be seen due to the change in the length scales. A better way of seeing the change in shock structure is by considering a minimum length scale for the shock which may be defined by

$$
w\left(\Delta_{1}, \Delta_{2}, \tau_{1}, \tau_{2}, \delta\right)=\left(\max _{\xi}\left(\frac{\mathrm{d} p}{\mathrm{~d} \xi}\right)\right)^{-1}
$$

Fig. 4 shows the variation of $w$ as a function of $\Delta_{1}$ for $\Delta_{2}=0.25$ with $\tau_{1}=1, \tau_{2}=0.01$ and $\delta=5 \times 10^{-6}$. The solid line marks the numeric solution and the dashed lines the asymptotic results for the three separate regimes. For $\Delta_{1}>0.5$ (Case I), the asymptotic results overlie the numeric results. As $\Delta_{1}$ decreases to less than 0.5 (Case II), a sudden decrease in the minimum shock width is seen, followed by a similar decrease when $\Delta_{1}$ decreases below 0.25 (i.e. $\Delta_{1}+\Delta_{2}<1 / 2$ ) corresponding to the appearance of the viscous inner shock (Case III).

The numeric and asymptotic methods described are now applied to parameter values relevant to sonic booms propagating through the atmosphere. For the particular case of air at $20^{\circ} \mathrm{C}$ at standard pressure

$$
\rho_{0}=1.2 \mathrm{~kg} \mathrm{~m}^{-3}, \quad c=343 \mathrm{~m} \mathrm{~s}^{-1}, \quad \beta=1.2 .
$$

Relaxation times are very sensitive to humidity. For a relative humidity of 50\%, the relaxation parameters associated with nitrogen (denoted by subscript 1), and oxygen (denoted by subscript 2) and the coefficient of thermoviscous diffusion are given by

$$
\begin{aligned}
& \tilde{\tau}_{1}=4.73 \times 10^{-4} \mathrm{~s}, \quad \tilde{\tau}_{2}=4.42 \times 10^{-6} \mathrm{~s}, \quad \frac{\tilde{\delta}}{c^{2}}=1.58 \times 10^{-10} \mathrm{~s}, \quad \frac{(\widetilde{\Delta c})_{1}}{c}=6.27 \times 10^{-5}, \\
& \frac{(\widetilde{\Delta c})_{2}}{c}=3.37 \times 10^{-4} .
\end{aligned}
$$




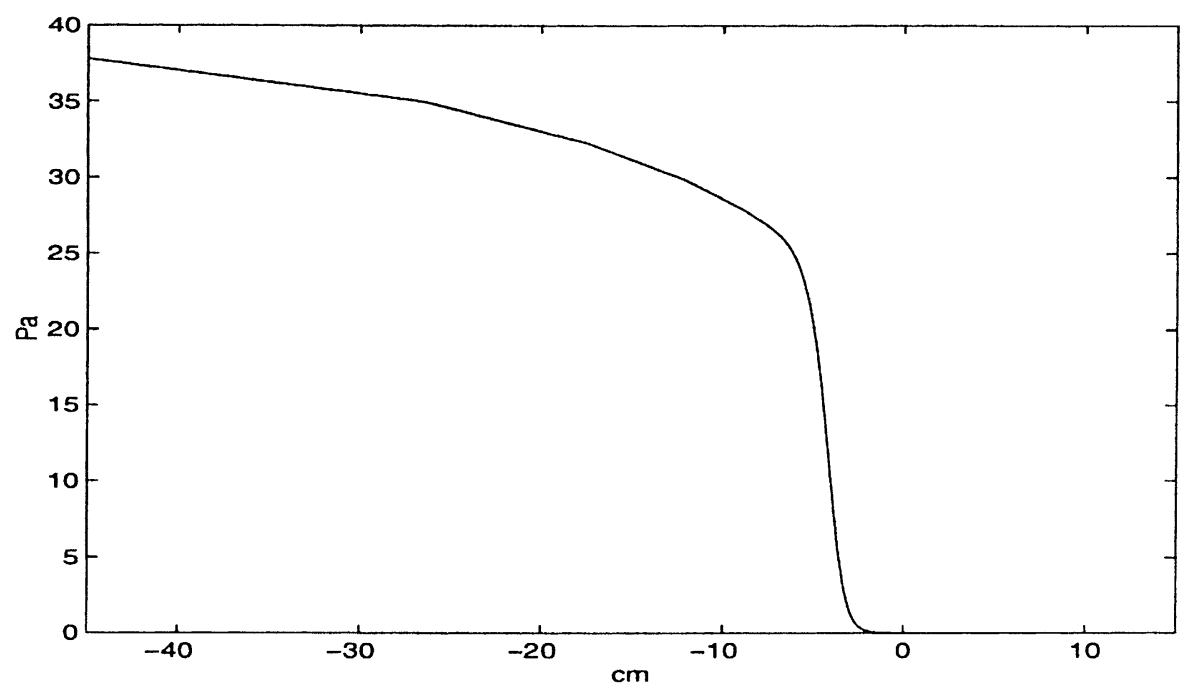

Fig. 5. Diagram shows the pressure profile in dimensional units, corresponding to the parameter values given by Kang and Pierce.

For convenience we choose the reference time introduced in the non-dimensionalisation (3) to be $t_{\text {ref }}=V \tilde{\tau}_{1}$, so $\tau_{1}=1$. For shock over pressures $\tilde{p}_{\text {sh }}$ less than $100 \mathrm{~Pa}, P_{\mathrm{sh}}<0.0005$ and $V$, the non-dimensional shock speed (7), is close to 1 . The relevant dimensionless parameters are then given by

$$
\tau_{1}=1, \quad \tau_{2}=0.0093, \quad \delta=\frac{0.0395 \mathrm{~Pa}}{\tilde{p}_{\mathrm{sh}}}, \quad \Delta_{1}=\frac{7.38 \mathrm{~Pa}}{\tilde{p}_{\mathrm{sh}}}, \quad \Delta_{2}=\frac{39.5 \mathrm{~Pa}}{\tilde{p}_{\mathrm{sh}}} .
$$

Hence the structure of the shock can be classified as one of the three separate cases depending on the value of the dimensional shock over pressure $\tilde{p}_{\text {sh }}$. For $\tilde{p}_{\text {sh }}<14.76 \mathrm{~Pa}$ we have Case I, a fully-dispersed shock of dimensional width $\mathrm{O}\left(c \tilde{\tau}_{1}\right)$ which corresponds to $\mathrm{O}(16 \mathrm{~cm})$.

For $14.76 \mathrm{~Pa}<\tilde{p}_{\text {sh }}<93.76 \mathrm{~Pa}$, we have Case II, with a sub-shock of width $\mathrm{O}(15 \mathrm{~mm})$. Finally, for $\tilde{p}_{\text {sh }}>$ 93.76 Pa we have Case III with a very narrow viscous shock, the width of which depends on $\tilde{p}_{\text {sh. }}$ Pierce and Kang [10] presented numerical data for the parameter values used above and $\tilde{p}_{\mathrm{sh}}=39.5 \mathrm{~Pa}$, though without plotting the resulting waveform. In Fig. 5 we plot the pressure profile in dimensional units, corresponding to the parameter values given by Kang and Pierce. Asymptotic results suggest that this should correspond to Case II, with a partly-dispersed solution controlled by the oxygen relaxation mode, together with a sub-shock of amplitude $\left(1-2 \Delta_{1}\right) \tilde{p}_{\text {sh }}=24.7 \mathrm{~Pa}$ controlled by the nitrogen relaxation mode. Numerical results using the method described in Section 4 are plotted in Fig. 5, where the shock structure described above can be clearly seen.

The results presented by Pierce and Kang [10] are within 5\% of the results of the present paper. Using the numerical method of Kang and Pierce, where $p$ is treated as the independent variable, $\xi$ as the dependent variable, and the typographical errors in their governing equations have been corrected, excellent agreement is obtained with the result using the method described in Section 4. No details were given by Kang and Pierce of the mesh size used in their calculations so it is likely that discrepancies are due to numerical error.

Thus for sonic boom propagation, asymptotic methods can be used to identify shock scales without the need for numerics. This is particularly useful when considering the more complicated problem of solving the governing equations (1), as a PDE rather than just considering travelling wave solutions. Numerics could be impractical for this problem, especially when considering very fine shock structures. It should be noted, however, that care must be taken when considering propagation through a realistic atmosphere. The molecular relaxation times are very sensitive to the amount of water vapour in the atmosphere, and hence, radically differ with altitude. Study is therefore needed 
to investigate the sonic boom waveform dependence on its own past history before comparisons can be made with numerics.

\section{References}

[1] G.B. Whitham, Linear and Nonlinear Waves, Wiley/Interscience, New York, 1974.

[2] M.J. Lighthill, Viscosity effects in sound waves of a finite amplitude, in: G.K. Batchelor, R.M. Davies (Eds.), Surveys in Mechanics, Cambridge University Press, Cambridge, 1956.

[3] J.D. Cole, On a quasi-linear parabolic equation occurring in aerodynamics, Quart. Appl. Math. 9 (1951) 225-236.

[4] E. Hopf, The partial differential equation $U_{t}+U U_{x}=U_{x x}$, Commun. Pure Appl. Math. 3 (1950) 201-230.

[5] J.F. Clarke, M. McChesney, Dynamics of Relaxing Gases, Butterworths, London, 1976.

[6] A.D. Pierce, Acoustics: An Introduction to its Physical Principles and Applications, McGraw-Hill, New York, 1981.

[7] P.A. Blythe, Nonlinear wave propagation in a relaxing gas, J. Fluid Mech. 37 (1969) 31-50.

[8] H. Ockendon, D.A. Spence, Nonlinear wave propagation in a relaxing gas, J. Fluid Mech. 39 (1969) 329-345.

[9] R. Cleveland, Propagation of sonic booms through a real, stratified atmosphere, Ph.D. Dissertation, The University of Texas at Austin, 1995.

[10] A.D. Pierce, J. Kang, Molecular relaxation effects on sonic boom waveforms, in: M.F. Hamilton, D.T. Blackstock (Eds.), Frontiers of Nonlinear Acoustics, Proceedings of the 12th ISNA, Elsevier, Amsterdam, 1990. 